\title{
Protagonismo Estudantil no Processo de Aprendizagem com Tecnologia: Uma Revisão Sistemática de Literatura
}

\author{
Elvis Medeiros de Melo, Neide Aparecida Alves de Medeiros, Nathalie Rose Ramos \\ da Fonseca Araújo, Edith Cristina da Nóbrega, Eduardo Henrique da Silva \\ Aranha, Marcia Jacyntha Nunes Rodrigues Lucena
}

Instituto Metrópole Digital - Universidade Federal do Rio Grande do Norte (UFRN) Av. Sen. Salgado Filho, 3000 - Lagoa Nova, CEP: 59.078-970 - Natal - RN - Brasil

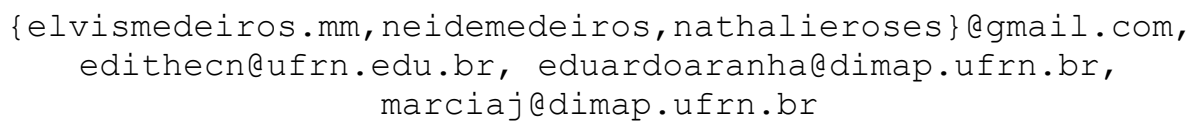

\begin{abstract}
Practices with technology use are becoming more widespread, but they often use it only to change the paradigm, the student remains passive in relation to his learning. This study aims to analyze the main works that put the student in the position of protagonist in the practices in educational computing, as well as the strategies that teachers are using to achieve this goal. We perform a systematic review of the literature available in Brazilian event annals and in Brazilian computer science journals in the last five years. Among the results, the main contributions that these studies have brought to this area of study are presented.
\end{abstract}

Resumo. Práticas com uso de tecnologia estão a cada dia sendo difundidas, mas muitas vezes a utilizam apenas para mudança de paradigma, o aluno continua sendo passivo em relação a sua aprendizagem. Este estudo tem o objetivo de analisar os principais trabalhos que colocam o aluno na posição de protagonista nas práticas em informática educacional, assim como as estratégias que os professores estão utilizando para atingir esse fim. Realizamos uma revisão sistemática da literatura disponível em anais de eventos brasileiros e em revistas brasileiras de informática na educação dos últimos cinco anos. Dentre os resultados, são apresentadas as principais contribuições que esses trabalhos trouxeram para essa área de estudo.

\section{Introdução}

As Tecnologias Digitais de Informação e Comunicação (TDICs) estão a cada dia mais presentes nas salas de aulas, trazidas pelos estudantes, e usadas por eles. Essas tecnologias são vistas como perturbadoras por parte de alguns profissionais da educação, que em algumas instituições chegam até a proibir o seu uso. Segundo Arruda et al (2017), esses recursos podem proporcionar o desenvolvimento de habilidades para transformar a informação obtida através das TDICs, constantemente em seu cotidiano, em conhecimento significativo. A proibição de seus usos não se sustenta.

Valente (2014) empreende em seu estudo sobre práticas pedagógicas com suporte de tecnologia, e alerta para a necessidade de as TDICs estarem bem relacionadas a essas práticas. Caso aconteça, pode ser um fator determinante para desenvolver maior motivação do aluno, através de atividades dinâmicas que favorecem 
VII Congresso Brasileiro de Informática na Educação (CBIE 2018)

Anais do XXIV Workshop de Informática na Escola (WIE 2018)

a constituição da criticidade, da participação, de ações inovadoras e posturas críticas quanto a sua realidade, desenvolvendo o protagonismo estudantil.

Podemos observar aspectos que ilustram o trabalho de Valente (2014) em Lima et al (2016). Eles discorrem sobre o uso do aplicativo Whatsapp com 10 alunos dos anos finais dos Ensino Fundamental, através de uma experiência m-learning. Os pesquisadores escolheram os dispositivos móveis e os aplicativos de desenho e apresentação de slides da Google. Utilizaram o smartphone para a captura de imagens por sugestão dos próprios estudantes. Segundo os autores, o smartphone possibilitou a realização das atividades colaborativas, além de incentivar os alunos a criar estratégias para o desenvolvimento das atividades de forma autônoma. Práticas como essa, apoiada por TDICs, colocam o aluno como protagonista de sua aprendizagem.

Arruda et al (2017) consideram o aluno como protagonista quando ele tem papel de sujeito ativo e participante do seu processo de aprendizagem. Ele consegue vivenciar e ultrapassar situações, funções e papéis que, em seu dia a dia, não tinha a oportunidade de exercer.

Práticas pedagógicas com suporte das TDICs, como em Lima et al (2016) estão se difundindo, colocando o aluno como centro do processo de aprendizagem. A falta de uma Revisão Sistemática de Literatura (RSL) que identifique se a literatura atual publicada nos últimos eventos da área de informática na educação, no cenário brasileiro, colocam o aluno na posição de protagonista de sua aprendizagem em práticas dessa área de estudo se faz necessária. A sua falta dificulta a adoção de práticas que colocam o aluno como protagonista por professores, além de embasar novas pesquisas no tema. Realizar essa RSL é o objeto de estudo do presente trabalho. Para tanto, delimitamos as perguntas sobre o tema segundo proposto por Kitchenham (2004), com a necessidade de uma RSL: Como as pesquisas atuais brasileiras acerca de práticas em informática educacional apontam ou direcionam para o aluno como sujeito ativo de sua aprendizagem? Que práticas estão sendo utilizadas? Responderemos essas perguntas no desenvolver da RSL.

Este artigo estrutura-se na presente introdução; seguido de metodologia utilizada na elaboração da revisão sistemática de literatura; seção de resultados, na qual discorremos sobre os trabalhos selecionados e extração dos dados; considerações sobre a RSL; e referências consultadas.

\section{Metodologia}

Adotamos a metodologia de Kitchenham (2004) para o desenvolvimento da RSL. Essa proposta apresenta alguns passos para a elaboração do trabalho, quais sejam: desenvolvimento do protocolo da RSL, incluindo a elaboração das questões da pesquisa em relação ao objetivo da RSL; seleção de estudos primários de acordo com o protocolo; análise de qualidade dos artigos encontrados; extração dos dados e síntese.

As questões da pesquisa foram assim delimitadas:

Q1: Como as pesquisas atuais brasileiras acerca de práticas em informática educacional apontam ou direcionam para o aluno como sujeito ativo de sua aprendizagem?

Q2: Que práticas estão sendo utilizadas?

Extraímos os dados de eventos e revistas brasileiras com o intuito de verificar o cenário brasileiro nas pesquisas em tais canais de divulgação científica. Acreditamos 
VII Congresso Brasileiro de Informática na Educação (CBIE 2018)

Anais do XXIV Workshop de Informática na Escola (WIE 2018)

que autores que fazem pesquisa na área de tecnologia educacional sempre procuram pelo menos um desses canais para divulgação de suas pesquisas científicas. São eles: Anais do Simpósio Brasileiro de Informática na Educação (SBIE), Workshop de Informática na Escola (WIE), Congresso sobre Tecnologias na Educação (Ctrl+E). Consideramos estes os principais eventos em Informática Educacional no Brasil, sendo este último um evento relativamente novo, mas com significante publicação de trabalhos relacionados à sala de aula. Selecionamos artigos das revistas de publicação científica: Revista Brasileira de Informática na Educação (RBIE) e a Revista Tecnologias na Educação (TecEdu), por considerar estas as duas principais revistas em Informática Educacional no Brasil. Buscamos por artigos datados entre 2014 a 2018, com o intuito de selecionar pesquisas mais recentes sobre estudos que colocam o aluno como protagonista nos processos de sua aprendizagem.

Para isso, utilizamos a seguinte String de busca nos canais: SBIE, WIE e RBIE.

\section{("aluno" OR "aprendiz" OR "sujeito ativo" OR "discente" OR "aprendente" OR "protagonista") AND ("processo de ensino e aprendizagem" OR "práticas educativas" OR "práticas educacionais")}

Os demais canais de busca (TecEdu e Ctrl+E) não dispunham de campos de busca automatizada. Nesses específicos, optamos por fazer a busca manual pela leitura do título do artigo e catalogando-os em uma planilha eletrônica colaborativa.

Definimos, segundo Kitchenham (2004), alguns critérios de inclusão e exclusão para serem usados na seleção de artigos nesta revisão sistemática de literatura:

Tabela 1. Critérios de Inclusão

\begin{tabular}{|c|c|}
\hline C-IN-1 & Práticas focadas no aluno \\
\hline C-IN-2 & Relatos de Experiência em Sala de Aula \\
\hline C-IN-3 & Trabalhos em Português \\
\hline
\end{tabular}

Tabela 2. Critérios de Exclusão

\begin{tabular}{|c|c|}
\hline C-OUT-1 & Formação de Professor \\
\hline C-OUT-2 & Desenvolvimento de Ferramentas e Soluções \\
\hline C-OUT-3 & Desenvolvimento de Metodologias \\
\hline C-OUT-4 & Revisões Sistemáticas de Literatura \\
\hline C-OUT-5 & Trabalhos em Língua Estrangeira \\
\hline C-OUT-6 & Trabalhos Duplicados \\
\hline C-OUT-7 & Análise de ferramentas e soluções \\
\hline
\end{tabular}

Os artigos também foram avaliados quanto à sua qualidade de escrita e possibilidades de contribuições para as questões do trabalho [Wazlawick 2014]. Seguindo as sugestões da literatura citada, classificamos os artigos em: Fortes, Médios e Fracos. Essa análise foi realizada a partir da leitura do títulos, seguidos pela leitura do abstract, além do uso da técnica de leitura rápida (scanning) do artigo.

Os artigos considerados como fortes tiveram uma análise aprofundada: foram realizados fichamentos através de uma planilha eletrônica compartilhada, enfatizando 
VII Congresso Brasileiro de Informática na Educação (CBIE 2018)

Anais do XXIV Workshop de Informática na Escola (WIE 2018)

seus pontos mais interessantes para a RSL, focando na resolução das questões Q1 e Q2. Verificamos quais artigos respondiam às perguntas levantadas.

Apresentamos algumas considerações a respeito dos trabalhos selecionados nas primeiras etapas de seleção dos artigos. A quantidade de artigos encontrados de acordo com os critérios de busca definidos são apresentadas na Tabela 3.

Tabela 3. Quantidade de artigos de acordo com a base de pesquisa selecionada

\begin{tabular}{|c|c|}
\hline Base & Quantidade de artigos encontrados \\
\hline Ctrl+E & 25 \\
\hline SBIE & 24 \\
\hline RBIE & 24 \\
\hline WIE & 13 \\
\hline TecEdu & 59 \\
\hline Total & $\mathbf{1 4 5}$ \\
\hline
\end{tabular}

Quantificamos os artigos quanto aos critérios de exclusão e por base pesquisada na tabela abaixo:

Tabela 4. Quantidade de artigos excluídos por base pesquisada e critério de exclusão

\begin{tabular}{|c|c|c|c|c|c|c|c|}
\hline Base & C-OUT-1 & C-OUT-2 & C-OUT-3 & C-OUT-4 & C-OUT-5 & C-OUT-6 & C-OUT-7 \\
\hline SBIE & 0 & 2 & 0 & 3 & 1 & 0 & 17 \\
\hline WIE & 3 & 1 & 1 & 1 & 0 & 0 & 4 \\
\hline Ctr+E & 1 & 2 & 2 & 3 & 0 & 0 & 4 \\
\hline TecEdu & 5 & 14 & 11 & 1 & 0 & 0 & 0 \\
\hline RBIE & 0 & 5 & 15 & 0 & 1 & 0 & 3 \\
\hline
\end{tabular}

Dos artigos que passaram pelo crivo dos critérios de inclusão e exclusão, consideramos apenas os fortes para análise dos dados. A quantidade de artigos oriundos do WIE foram: Fortes - 3, os demais foram excluídos na etapa de seleção de literatura; SBIE: Todos foram excluídos na etapa de seleção de literatura; Ctrl+E: Fortes - 6, Médios - 3, Fracos - 1; TecEdu: Fortes - 10, Médios - 13, Fracos - 12; e RBIE: Fortes 1, os demais foram excluídos na etapa de seleção de literatura. Analisamos apenas os artigos considerados fortes e que passaram pelos critérios de exclusão.

Dos trabalhos primários selecionados como relevantes [Kitchenham 2004], identificamos para as análises realizadas abaixo:

Tabela 5. Trabalhos selecionados para a revisão sistemática de literatura

\begin{tabular}{|c|l|l|l|}
\hline ID & Base & \multicolumn{1}{|c|}{ Autor(es) } & \multicolumn{1}{|c|}{ Título } \\
\hline A1 & TecEdu & Pereira e Almeida (2014) & $\begin{array}{l}\text { Nativos Digitais na Educação Infantil: Os desafios } \\
\text { Pedagógicos de lidar com as Tecnologias dentro e fora da } \\
\text { Escola. }\end{array}$ \\
\hline A2 & TecEdu & Almeida e Santos (2015) & Realidade Aumentada na Educação \\
\hline
\end{tabular}


VII Congresso Brasileiro de Informática na Educação (CBIE 2018)

Anais do XXIV Workshop de Informática na Escola (WIE 2018)

\begin{tabular}{|c|c|c|c|}
\hline $\mathrm{A} 3$ & TecEdu & Ruas e Lima (2015) & $\begin{array}{l}\text { Interdisciplinaridade e Aprendizagem Significativa com o } \\
\text { Uso de Tecnologias Digitais }\end{array}$ \\
\hline A4 & TecEdu & Lopes e Araújo (2016) & $\begin{array}{l}\text { Uso de Tecnologias Digitais na Escola: Uma Visão das } \\
\text { Crianças do Quinto Ano do Ensino Fundamental }\end{array}$ \\
\hline A5 & TecEdu & $\begin{array}{l}\text { Tenório, Nascimento e } \\
\text { Tenório (2017) }\end{array}$ & $\begin{array}{l}\text { Uso do Software GeoGebra, Blog, e-mail e Whatsapp no } \\
\text { Estudo de Matemática }\end{array}$ \\
\hline A6 & TecEdu & $\begin{array}{l}\text { Silva, Rodrigues e Silva } \\
\text { (2017) }\end{array}$ & $\begin{array}{l}\text { Produção de Vídeos Educativos Com o Aparelho de } \\
\text { Telefone Celular: Uma Proposta para Promover a } \\
\text { Aprendizagem Significativa no Ensino de Ciências no } \\
\text { Ensino Fundamental }\end{array}$ \\
\hline A7 & WIE & $\begin{array}{l}\text { Martins, Reis e Marques } \\
(2016)\end{array}$ & $\begin{array}{l}\text { Inserção da programação no ensino fundamental Uma } \\
\text { análise do jogo Labirinto Clássico da Code.org através de } \\
\text { um modelo de avaliação de jogos educacionais }\end{array}$ \\
\hline A8 & WIE & Reis et al (2017) & $\begin{array}{l}\text { Pensamento Computacional: Uma Proposta de Ensino com } \\
\text { Estratégias Diversificadas para Crianças do Ensino } \\
\text { Fundamental }\end{array}$ \\
\hline A9 & WIE & Teixeira et al (2015) & $\begin{array}{l}\text { Programação de computadores para alunos do ensino } \\
\text { fundamental: A Escola de Hackers }\end{array}$ \\
\hline A 10 & $\mathrm{Ctrl}+\mathrm{E}$ & $\begin{array}{l}\text { Caitano, Azevêdo e Viana } \\
\text { (2016) }\end{array}$ & $\begin{array}{l}\text { Letramento digital no ensino de língua portuguesa em uma } \\
\text { escola do semiárido potiguar: os jogos educativos na } \\
\text { mediação do ensino-aprendizagem }\end{array}$ \\
\hline A11 & $\mathrm{Ctrl}+\mathrm{E}$ & Falcão (2016) & $\begin{array}{l}\text { Integrating Aspects of Gamification in the Classroom: } \\
\text { Takeaways from a Tentative Experience with } \\
\text { Undergraduates }\end{array}$ \\
\hline A12 & $\mathrm{Ctrl}+\mathrm{E}$ & $\begin{array}{l}\text { Bezerra, Figueiredo, Camelo } \\
\text { Filho (2016) }\end{array}$ & $\begin{array}{l}\text { A Construção Do Conhecimento Colaborativo No } \\
\text { Ciberespaço: Uma Análise Das Trocas Comunicativas Do } \\
\text { Grupo Xenserver No Aplicativo Telegram }\end{array}$ \\
\hline A13 & $\mathrm{Ctrl}+\mathrm{E}$ & Caitano, Azevêdo (2016) & $\begin{array}{l}\text { Oficina Robótica Pedagógica Livre: um instrumento de } \\
\text { multidisciplinaridade }\end{array}$ \\
\hline A14 & $\mathrm{Ctrl}+\mathrm{E}$ & Bezerra et al (2016) & $\begin{array}{l}\text { O Desenvolvimento da Tipologia Dissertativa no Ensino } \\
\text { Médio em meio à Interação OnLine }\end{array}$ \\
\hline A15 & $\mathrm{Ctrl}+\mathrm{E}$ & Hitzschky et al (2016) & $\begin{array}{l}\text { Práticas Educativas com o de Dispositivos Móveis em } \\
\text { Aulas de Campo: Aprendizagem Para Além dos Muros da } \\
\text { Escola }\end{array}$ \\
\hline A16 & RBIE & $\begin{array}{l}\text { Alves, Sampaio e Elia } \\
(2014)\end{array}$ & $\begin{array}{l}\text { DuinoBlocks: Desenho e Implementação de um Ambiente } \\
\text { de Programação Visual para Robótica Educacional. }\end{array}$ \\
\hline A17 & TecEdu & Costa e Pessoa (2014) & $\begin{array}{l}\text { A inserção de um indivíduo na cultura digital: o papel da } \\
\text { escola neste contexto }\end{array}$ \\
\hline A18 & TecEdu & $\begin{array}{l}\text { Grimaldi, Pereira e Santos } \\
(2015)\end{array}$ & $\begin{array}{l}\text { Uma proposta colaborativa no ensino de matemática por } \\
\text { meio de construção de vídeos }\end{array}$ \\
\hline A19 & TecEdu & $\begin{array}{l}\text { França, Reategui e Collares } \\
(2016)\end{array}$ & $\begin{array}{l}\text { Engajamento dos Alunos para Colaboração em um Ambiente } \\
\text { utilizando a Aprendizagem baseada em Projetos e a } \\
\text { Gamificação }\end{array}$ \\
\hline
\end{tabular}


VII Congresso Brasileiro de Informática na Educação (CBIE 2018)

Anais do XXIV Workshop de Informática na Escola (WIE 2018)

\begin{tabular}{|l|l|l|l|}
\hline A20 & TecEdu & Chaparro (2016) & $\begin{array}{l}\text { O Uso do Facebook e do Blog Como Ferramentas no } \\
\text { Desenvolvimento de Projeto Interdisciplinar para o Ensino } \\
\text { Médio }\end{array}$ \\
\hline
\end{tabular}

\section{Resultados e Discussões}

A seguir, procuramos responder as perguntas delimitadas pela revisão sistemática de literatura segundo a análise dos artigos selecionados [Kitchenham 2004]. Citaremos em ordem categórica e sistemática, de acordo com a identificação presente na Tabela 4.

\subsection{Como as pesquisas atuais brasileiras acerca de práticas em informática educacional apontam ou direcionam para o aluno como sujeito ativo de sua aprendizagem?}

Os autores de A1, durante suas aulas, deixam que as crianças brinquem com um determinado jogo, software ou aplicativo e, em seguida, sentam em roda e, através da mediação da professora, conversam entre si sobre definições, utilidades e o sentido do uso da tecnologia que acabara de usar. As falas das crianças são registradas através de gravação de voz no celular e de desenhos que elas mesmas criam. Já em A4, percebeuse que as crianças ainda não têm muita clareza das possibilidades que as tecnologias digitais podem lhes oferecer em relação a aprendizagem. Percebeu-se também que as tecnologias digitais são utilizadas com mais intensidade fora das instituições de ensino e que, para as crianças, o primeiro contato com elas geralmente é no meio familiar. As crianças afirmaram utilizá-las tanto para o processo de aprendizagem como para o seu entretenimento.

Os autores de A2 creditam o uso de Realidade Aumentada (RA) como facilitadora do processo de trabalhar situações onde o professor precisaria ilustrar desenhos no quadro, mas que muitas das vezes não fazia sentido aos alunos. A RA possibilitou também a prática dentro de sala de aula sem a necessidade de locomoção. Para A6, a produção de vídeos com uso do smartphone pode ser usado como instrumento potencialmente capaz de ampliar a capacidade de ensinar e aprender. Apesar de A16 versar sobre o desenho e implementação de um ambiente com linguagem de programação visual, reconhece, de modo sutil, a condição do aluno como protagonista de seu aprendizado. Tal apontamento é percebido a partir do momento em que apresenta os benefícios da robótica educacional para o desenvolvimento da aprendizagem.

Em A3, os autores identificaram a interação profícua entre os estudantes e a autonomia que tiveram na etapa de pesquisas na internet e em livros. A busca por informações e a organização foram salutares, pois, ao levantar novos assuntos e relacioná-los à temática geral proposta pelo projeto, os estudantes qualificaram-se para as discussões intergrupos e posteriormente entre os grupos, mediados pelas professoras envolvidas no projeto. Para os alunos, segundo A5, as tecnologias usadas na pesquisa, GeoGebra, blog, whatsapp e e-mail, foram capazes de ajudar no desenvolvimento das atividades de Matemática. Tais tecnologias aguçaram a curiosidade, despertaram o interesse e motivam o aprendizado. A inserção de tecnologias de forma integrada como proposto na abordagem de ensino foi uma forma positiva de aproximação entre alunos e com o professor, além de despertar o interesse e motivar. Os alunos enxergaram os recursos como benéficos, porém, alguns acharam as atividades trabalhosas.

Em A20, os autores apresentam uma experiência desenvolvida em sala de aula em um projeto interdisciplinar com o uso de ferramentas como o Facebook e blog. O 
VII Congresso Brasileiro de Informática na Educação (CBIE 2018)

Anais do XXIV Workshop de Informática na Escola (WIE 2018)

desenvolvimento da atividade se deu de maneira colaborativa e foi possível perceber que o projeto descrito foi pensado para que os alunos fossem realmente atuantes quanto a sua aprendizagem, e o professor atuasse como um mediador do processo. A experiência relatada no artigo aponta para o aluno como protagonista (sujeito ativo) de sua aprendizagem por apresentar que nas fases de desenvolvimento do projeto interdisciplinar proposto, os alunos tiveram a autonomia e a autoria estimulada e alavancada em vários aspectos, como a definição do tema, em discussão entre os alunos; a divisão dos grupos que também partiu deles; a criação e publicação de posts para o blog. Além de uma autoavaliação individual e outra global (da turma). E, por fim, o professor atuando na condição de orientador e avaliador.

O estudo realizado em A7 demonstrou que o jogo trouxe uma ótima experiência aos usuários, alinhando motivação e aprendizagem. O impacto alcançado pelos objetivos de aprendizagem do jogo demonstra que a maioria dos alunos não possuía experiência prévia com os conceitos abordados, ainda assim indicaram aumento em seu conhecimento sobre programação após o uso da ferramenta. As estratégias adotadas em A8 colocam o aluno no centro de seu aprendizado. O ensino de conceitos da Ciência da Computação e programação de computadores em jogos foi abordado nesta experiência por meio de metodologias diversificadas. Já os autores de A9 relatam o processo de criação do Projeto Escola de Hackers e seus desdobramentos com o objetivo de oportunizar um espaço para o desenvolvimento de competências na área de programação de computadores e de raciocínio lógico matemático tanto para os estudantes como para os professores.

A10 relata que com a utilização dos jogos digitais, os educandos tornaram-se protagonistas do seu próprio conhecimento, respeitando e seguindo o seu ritmo de aprendizagem de forma prazerosa. A metodologia de gamificação foi abordada também pelos autores de A11, com a ideia é trazer contextos de aprendizagem mais perto do mundo dos jogos tornando o aprendizado divertido e envolvente para a atual geração de estudantes, e com isso um impacto positivo na sua realização e construção do conhecimento. Os jogos digitais estão entre os passatempos favoritos das crianças e dos jovens nos dias de hoje, provocando uma motivação intrínseca procurado pelos educadores. Percebe-se em A19 que, mesmo em se tratando do engajamento de alunos de maneira colaborativa, sobressai ao foco, a análise de dados com a finalidade de avaliação do uso de metodologias inovadoras como Aprendizagem baseada em Projetos e a Gamificação em Ambiente Virtual de Aprendizagem. Nota-se que o trabalho busca, a seu modo, destaca a importância do trabalho colaborativo e o engajamento dos alunos para evolução de sua aprendizagem.

A12 revela o aprendizado em rede através de trocas comunicativas de textos, imagens e links em um grupo de usuários avançado por meio do aplicativo Telegram. As práticas comunicativas do grupo são comparadas a comunidades de prática. As interações fazem com que os alunos reflitam sobre os temas debatidos e se tornem protagonistas da sua aprendizagem. O domínio da língua escrita impulsionou a produção de texto em contexto digital e A14. Os alunos tornaram-se sujeitos ativos na construção do conhecimento e motivados para elaborar e aprimorar os textos que foram construídos online. Já em A17, um aluno é tomado como base para análise, com isso, ele torna-se, em parte, um sujeito protagonista, uma vez que suas experiências e práticas são absorvidas como fonte de análise para reflexão do papel do professor e do currículo. A partir de um estudo de caso, com um aluno específico, são considerados alguns apontamentos para que o currículo e a atuação do professor contribuam diretamente para a inserção da tecnologia no cotidiano escolar. 
VII Congresso Brasileiro de Informática na Educação (CBIE 2018)

Anais do XXIV Workshop de Informática na Escola (WIE 2018)

Em A13 os alunos foram estimulados a criarem e buscarem resultados para situações problemas e incentivados a trocar conhecimentos entre eles por intermédio de uma oficina de robótica produzida com materiais recicláveis. A oficina também trabalhou a sustentabilidade incentivando a reciclagem, transformando o que os outros não querem mais em instrumentos educativos multidisciplinares. A15 relatou a importância de discutir o ambiente escolar de uma maneira lúdica e interativa, no qual, o aluno pesquisou e propôs soluções para melhorar o espaço escolar.

Em A18 foi possível perceber a consistente atuação do aluno como sujeito ativo de sua aprendizagem, pois foi proposto que os alunos produzissem vídeos-aulas contendo explicações de exercícios e postassem em um blog para que, de maneira colaborativa, fosse promovida a aprendizagem entre os participantes da proposta. Além disso, para reforçar a importância do aluno como protagonista de sua aprendizagem é apresentado, ao final, uma avaliação da atividade na visão do aluno. Entende-se que os sujeitos envolvidos nesta proposta tiveram autonomia no processo criativo e puderam reconhecer, ao final, de maneira autoavaliativa, as significações da atividade proposta. Pois a produção de vídeo-aula e a postagem em blog (divulgação) faz com que o aluno aprenda, de fato, fazendo, tornando o processo muito mais significativo, por se reconhecerem como peças importantes para o seu aprendizado e dos colegas em mesma condição, considerando, desse modo, o perfil colaborativo.

\subsection{Que práticas estão sendo utilizadas?}

Em A1 não há um planejamento fixo e devidamente sistematizado dos momentos de aprendizagem que ocorrem diariamente. O conhecimento é construído a partir das temáticas abordadas pelas crianças e observadas pelos professores, desde o primeiro momento em que a criança entra na escola. Há necessidade de compreender o tipo de contato que as crianças costumam ter com aparatos como celular, tablet, computador, notebooks e Internet. $\mathrm{O}$ uso de dispositivos móveis propiciou a investigação do ambiente escolar e do seu entorno em A15. Os alunos detectaram os problemas e enxergaram soluções, melhorando aspectos como: argumentação, criticidade e responsabilidade com o ambiente.

Já em A3, a aprendizagem significativa dos estudantes, acerca de temática proposta, tendo como ferramenta pedagógica as tecnologias digitais para pesquisas na internet e produção dos mapas conceituais online. A proposta de A20 é o desenvolvimento de um projeto interdisciplinar utilizando ferramentas como o Facebook e o blog, colocando o aluno como protagonista de sua aprendizagem, uma vez que ele decide, atua e reflete sobre suas ações, tendo o professor como peça mediadora do processo, quanto a orientação e a avaliação.

O uso software GeoGebra e blogs no estudo de função polinomial do $1^{\circ}$ grau em foi protagonista em A5. Houve manuseio do GeoGebra para aprender os conceitos de função do $1^{\text {o }}$ grau e exercitá-los; escolha de uma situação-problema e criação de estratégias de solução com o GeoGebra; elaboração de um blog com a narrativa do problema e o passo a passo da resolução; estudo dos blogs dos colegas e reproduzir as atividades no GeoGebra. Na comunicação extraclasse, e-mail e whatsapp foram utilizados.

Os alunos do sexto e sétimo ano da Escola Municipal Anne Frank (Palmas TO) estão produzindo vídeos educativos a partir dos conceitos trabalhados em sala, durante as aulas de Ciências, segundo A6. A produção de vídeo aulas em A18 contendo 
VII Congresso Brasileiro de Informática na Educação (CBIE 2018)

Anais do XXIV Workshop de Informática na Escola (WIE 2018)

explicações de exercícios pelos próprios alunos, a postagem em um blog e a autoavaliação dos alunos protagonistas da atividade desenvolvida.

Os autores de A10 resolveram propor para os alunos do $8^{\circ}$ ano do ensino fundamental uma oficina com jogos digitais, intitulada de Letramento Digital no Ensino de Língua Portuguesa. Esta oficina foi aplicada em uma escola estadual, na cidade de Angicos/RN, e abordou os jogos: KHangMan, Roda a Roda, Soletrando e por fim o Jogo da Acentuação (adequado ao novo acordo ortográfico). O relato de A7 avaliou a aplicação da plataforma Code.org, especificamente do jogo Labirinto Clássico, que aborda conceitos básicos relacionados à lógica de programação utilizando cenários do Angry Birds, Plants vs Zombies e A Era do Gelo.

Em A8, metodologias como gamificação, computação desplugada, Storytelling e Aprendizagem Significativa são adotadas para desenvolver importantes habilidades do pensamento computacional nos alunos do $8^{\circ}$ e $9^{\circ}$ anos do ensino fundamental. Os alunos trabalharam tanto individualmente como em grupos em momentos distintos de um curso que teve a duração de seis meses e uma carga horária de 30 horas. Já o A9 utilizou o software Scratch para oportunizar o aprendizado de técnicas e habilidades de programação de computadores para alunos do $6^{\circ}$ ao $9^{\circ}$ anos do Ensino Fundamental. Nesta prática os alunos conhecem o ambiente do Scratch, os comandos necessários para a programação e por fim os alunos podem criar seus próprios jogos. A linguagem de programação escolhida para esta abordagem foi o Scratch e uma placa de Arduíno UNO contribuiu para promover a criatividade e a capacidade de resolução de problemas nesta experiência com 20 crianças que ocorreu em uma escola municipal no interior do Rio Grande do Norte. Em contrapartida, A19 desenvolve de um projeto de um Loja Virtual em Ambiente Gamificado proposto pelo professor. Questionários com os alunos sobre o desenvolvimento da proposta e registro das ações dos alunos durante a execução do projeto no Ambiente Gamificado. Tudo isso, gerando dados para análise (avaliação), que é o foco do trabalho. Uma oficina de robótica livre foi o instrumento de multidisciplinaridade abordado em A13.

A11 relata que graduandos do departamento de Ciência da Computação de uma universidade pública brasileira, foram divididos em grupos para projetar e prototipar um sistema (aplicativo móvel, jogos digitais, web site, ou outro) para apoiar a aprendizagem de um conteúdo de sua escolha, alinhado com a teoria de aprendizagem (de sua escolha). Já os autores de A2 propõem atividades por meio do Software Aumentaty Author, e aplicado pelo professor a 29 (alunos) da 8 a Série do Ensino Fundamental. Posteriormente, os alunos eo professor responderam questionários distintos, em cenários antes e após a utilização da realidade aumentada.

O uso do Telegram para construir o conhecimento colaborativo no ciberespaço foi destaque em A12 para compreender como se dá a criação e a interatividade de um grupo na cibercultura. Em A14, os alunos utilizaram o Google Drive para elaborarem textos dissertativos de maneira colaborativa, expandindo conhecimentos e refletindo sobre a escrita.

\section{Considerações Finais}

No cenário brasileiro, podemos observar um ambiente promissor de pesquisas que abordam o protagonismo estudantil em práticas com uso de tecnologias nos artigos selecionados para essa RSL. No entanto, apesar dos títulos e/ou abstracts sugerirem uma aprendizagem centrada no aluno, dos 145 artigos analisados 20 destes foram avaliados como fortes ou médios por sua contribuição textual para a RSL e 105 foram 
excluídos para esta revisão por não terem conexão com o protagonismo estudantil, o que revela uma eliminação direta de $72,41 \%$ dos artigos selecionados. Saindo desse padrão, dentre os textos consultados, a revista TecEdu figura como uma boa fonte de pesquisa com práticas voltadas para os alunos, nos fornecendo $50 \%$ dos trabalhos selecionados.

Entre os recursos mais utilizados em sala de aula, merece destaque o uso do celular em práticas que favorecem e transformam o aluno como protagonista, além de recursos que podem ser instalados nele, como Whatsapp, Telegram, além da câmera dos próprios dispositivos, usados também para a compreensão de como estão sendo utilizados pelos alunos. O uso do blog, assim como de jogos educacionais tiveram destaque nas pesquisas selecionadas. O Scratch, assim como outros recursos digitais estão sendo utilizados para o desenvolvimento do pensamento computacional, bem como o GeoGebra para a aprendizagem e protagonismo estudantil em práticas de construção matemática.

Por fim, um fato interessante a ser observado é a quantidade de artigos selecionados dos últimos dois anos. Isso mostra que práticas que oportunizam o protagonismo estudantil com suporte de tecnologia estão crescendo e estão sendo difundidas. Diante disso, pretende-se expandir as análises desta RSL, incluindo novos critérios de inclusão, além de busca em outros canais de divulgação brasileiros de pesquisa e práticas em informática educacional.

\section{Referências}

Arruda, J. S. et al (2017) Tecnologias digitais e o processo de protagonismo estudantil no Ensino Fundamental. In: VI Congresso Brasileiro de Informática na Educação (CBIE 2017). Anais do XXIII Workshop de Informática na Escola (WIE 2017).

Kitchenham, B. A. (2004). Procedures for undertaking systematic reviews. Technical report, Computer Science Department, Keele University.

Lima, M. S. S.; Lima, L. L. V.; Lima-Neto, C. S.; Castro-Filho, J. A. (2016). Experiência de Aprendizagem Móvel: o uso do WhatsApp no Ensino Fundamental. In: V Congresso Brasileiro de Informática na Educação (CBIE 2016). Anais dos Workshops do V Congresso Brasileiro de Informática na Educação (WCBIE 2016).

Valente, J. A. (2014) Aprendizagem ativa no ensino superior: A proposta da sala de aula invertida. Disponível em: $<$ https://goo.gl/Fmm4Jv>. Acesso em: 01 jun. 2018 .

Wazlawick, R.S. (2014) Metodologia de pesquisa para ciência da computação. 2.ed. Rio de Janeiro: Elsevier. 